Fluctuation and Noise Letters

Vol. 4, No. 3 (2004) L?

(C) World Scientific Publishing Company

\title{
Stochastic phase-field simulations of symmetric alloy solidification
}

\author{
R. Benítez and L. Ramírez-Piscina \\ Department de Física Aplicada, Universitat Politècnica de Catalunya \\ Doctor Marañón 44, E-08028 Barcelona, Spain.
}

Received (8 June 2004)

Revised (30 July 2004)

Accepted (5 August 2004)

\begin{abstract}
We study initial transient stages in directional solidification by means of a non-variational phase field model with fluctuations. This model applies for the symmetric solidification of dilute binary solutions and does not invoke fluctuation-dissipation theorem to account for the fluctuation statistics. We devote our attention to the transient regime during which concentration gradients are building up and fluctuations act to destabilize the interface. To this end, we calculate both the temporally dependent growth rate of each mode and the power spectrum of the interface evolving under the effect of fluctuations. Quantitative agreement is found when comparing the phase-field simulations with theoretical predictions.
\end{abstract}

Keywords: Phase-field models; fluctuations; solidification; transient stages.

\section{Introduction}

Solidification processes constitute a primary subject of research in materials science. One main objective is to achieve the prediction and control of the final macroscopic properties (mechanical, electrical, etc) of solids grown from their melts. These properties largely depend on the microstructure, which in turn is result of the conditions at which the solid was grown [1]. For example, relevant features at the microscopic scale such as compositional inhomogeneities or the presence of grain boundaries are direct consequences of instabilities and subsequent dynamics of the solidification front. Of particular interest for the metallurgical community has long been the directional solidification of mixtures and alloys, of direct relevance in processes of zone melting and for the Bridgman method [2]. This configuration is also an archetypical model system in nonlinear physics to study pattern selection and complex dynamics [3]. In this context it is known that the final wavelength of the dendritic array depends on the history [4], and specifically on the initial destabilization of the solidification front $[5,6]$. This is a non-steady noise amplification process occurring during the early solute redistribution transients, for which considering both fluctuations and transient effects are of key importance. Experimental work 
in this regime can be found in $[7,8]$. We are interested here in dealing with these aspects in numerical simulations of a directional solidification experiment, by using a phase field model supplemented with fluctuations.

During the last decade, the phase-field model has emerged as a quantitative simulation technique to study complex interfacial morphologies [9]. Recently, the phase field approach has deserved much attention because it can easily incorporate effects like system anisotropies, kinetic attachment or equilibrium fluctuations which might be difficult to include with other simulation techniques. This method avoids the tracking of the moving front by introducing a continuous order parameter $\phi(\vec{r}, t)$ (the phase field), which takes different constant values at the bulk phases separated by a smooth interface of width $W$. The model then consists in a set of coupled equations for $\phi(\vec{r}, t)$ and for the diffusion field which drives the interfacial dynamics, and that are constructed so that it reproduces the physical dynamics in the limit $W \rightarrow 0$. Early formulations of phase field models were variational, i.e. the model equations were derived from functional derivatives of a single free energy functional, but the convergence of such models was rather poor. Recent advances in phase field formulations, either based in the so called thin interface limit $[10,11]$ or in higher order expansions [12], permitted to improve dramatically the convergence of the model, while dealing with some specific realistic situations.

However the standard procedure to introduce fluctuations in phase field models is restricted to variational formulations [13-15], analogous to the model $\mathrm{C}$ of critical dynamics [16-18], where the intensity of the fluctuations can be determined by using a fluctuation-dissipation relation. As the more recent phase field formulations do not maintain this variational structure, the fluctuation-dissipation relation cannot be used to infer the statistics of the noise appearing in the equations. In the model and simulations presented here, we rely on a recent calculus [19] that projects the dynamics of a generic stochastic phase field model to the motion of the fluctuating interface. This procedure provides a prescription for the intensity of the noise terms in the model, accounting for fluctuations of both internal and external origin. This approach has previously been used in equilibrium situations only [19], and our aim here is to employ it in a transient, out of equilibrium, situation. We will restrict ourselves to the study of the initial destabilization in the linear regime, where comparison with theory is possible, as a first step to the complete problem of considering the whole competition process in the presence of fluctuations. The validation of this approach should be also useful in other situations of interfacial dynamics with fluctuations, both in non variational formulations of phase field models and in considering more general sources of fluctuations, of external origin.

\section{Stochastic Phase-Field Model for Symmetric Directional Solidifica- tion}

In a directional solidification experiment, the mixture sample is pulled at a velocity $\tilde{v}_{p}$ in an externally imposed temperature gradient given by $T(\tilde{z})=T_{M}+\tilde{G} \tilde{z}$, where $T_{M}$ is the melting temperature of the mixture and the tilde refers to physical units. For pulling velocities higher than a certain critical value, the interface becomes unstable [20] giving rise to cellular regimes. The diffusive field which drives the interface dynamics is the reduced solute concentration field $u=\frac{C-C_{\infty}}{\Delta C_{0}}$, where $C_{\infty}$ 
is the solute concentration of the sample in the liquid bulk far from the solid-liquid interface and $\Delta C_{0}=C_{L}^{0}-C_{S}^{0}$ is the miscibility gap which is approximated to be constant in this model. We are interested in the symmetric case for which the diffusion coefficients in both phases are equal, situation which is typical in dilute mixtures of liquid crystals. We consider a modified version of a non-variational phase-field model presented by Karma $[11,21]$ for the directional solidification of a symmetric dilute alloy. Scaling space and time with $l=D / \tilde{v}_{p}, \gamma=l^{2} / D$, being $D$ the solutal diffusivity, we obtain the phase-field equations

$$
\begin{aligned}
\alpha \varepsilon^{2} \partial_{t} \phi & =\varepsilon^{2} \nabla^{2} \phi-f^{\prime}(\phi)-\varepsilon \lambda g^{\prime}(\phi)\left(u+\frac{z-t}{l_{T}}\right)+\varepsilon^{\frac{3}{2}} \eta(\mathbf{r}, t) \\
\partial_{t} u & =\nabla^{2} u+\frac{1}{2} \partial_{t} h(\phi)-\nabla \cdot \mathbf{q}(\mathbf{r}, t)
\end{aligned}
$$

with

$$
\begin{aligned}
\left\langle\eta(\mathbf{r}, t) \eta\left(\mathbf{r}^{\prime}, t^{\prime}\right)\right\rangle & =2 \sigma_{\phi}^{2} \delta\left(\mathbf{r}-\mathbf{r}^{\prime}\right) \delta\left(t-t^{\prime}\right) \\
\left\langle q_{i}(\mathbf{r}, t) q_{j}\left(\mathbf{r}^{\prime}, t^{\prime}\right)\right\rangle & =2 \sigma_{u}^{2} \delta_{i j} \delta\left(\mathbf{r}-\mathbf{r}^{\prime}\right) \delta\left(t-t^{\prime}\right) .
\end{aligned}
$$

In the last equations, $\varepsilon=W / l$ stands for the dimensionless thickness of the interface, and $l_{T}=m \Delta C_{0} / G$ is the thermal length imposed by the external gradient, being $m$ the liquidus slope of the alloy. Fluctuations have been included following the procedure introduced in [19] to account for the equilibrium statistics. It can be shown that the model defined by Eqs. (11), (2), (3) and (4) recovers the classical moving boundary description of the problem in the limit of $\varepsilon \rightarrow 0$ (small interface width) and $\sigma_{\phi}, \sigma_{u} \ll 1$ (small noise intensities). For this particular model, and performing a matching asymptotic procedure up to first order in $\varepsilon$, we find the relations $\lambda=\frac{I_{1}}{I_{2}} \frac{1}{d_{0}}, \alpha=\frac{\beta+I_{4} \varepsilon}{d_{0}}, \sigma_{\phi}^{2}=\frac{\beta v_{m} I_{1}}{d_{0}^{2} \Delta C_{0}(1-k) N_{A} l^{d}}$, and $\sigma_{u}^{2}=\frac{C_{L}^{0} v_{m}}{N_{A} \Delta C_{0}^{2} l^{d}}$, where $I_{1}$, $I_{2}$ and $I_{4}$ are integral constants given by $I_{1}=2 \sqrt{2} / 3, I_{2}=16 / 15$ and $I_{4}=0.55$, and we have chosen the particular set of functions $f(\phi)=-\frac{1}{2} \phi^{2}+\frac{1}{4} \phi^{4}, g(\phi)=$ $\phi-\frac{2}{3} \phi^{3}+\frac{1}{5} \phi^{5}$ and $h(\phi)=\phi$. In the last relations, $\beta$ is the kinetic attachment, $k$ the segregation coefficient, $N_{A}$ the Avogadro's number, $v_{m}$ the molar volume and $d_{0}=\sigma T_{M} / L m \Delta C_{0}$ the chemical capillary length, where $\sigma$ is the surface energy and $L$ the latent heat per unit volume.

\section{Simulation Results}

As a quantitative benchmark of the results presented in [19], we perform numerical simulations of the phase-field model and compare them with theoretical predictions obtained from the moving boundary description of the front. We will present results for both the stationary power spectrum of the interfacial fluctuations and for the wavelength selection during the initial recoil transient.

The substance parameters correspond to the liquid crystal 4-n-octylcyanobiphenil (8CB), which has been used in several quantitative experimental works $[7,22,23]$. The substance parameters have been obtained from [7,22,23], and are given by $k=0.9, m=88.46 \mathrm{~K} / \mathrm{mol}, T_{M}=313.5 \mathrm{~K}, \sigma=2.2 \times 10^{-4} \mathrm{~J} / \mathrm{m}^{2}, \beta=113.04$ $\mathrm{s} \mathrm{mol} / \mathrm{m}, D=4 \times 10^{-10} \mathrm{~m}^{2} / \mathrm{s}, L=2.2 \times 10^{6} \mathrm{~J} / \mathrm{m}^{3}, Z=291.44 \mathrm{~g}$ (molecular weight) and $\rho=10^{6} \mathrm{~g} / \mathrm{m}^{3}$ (density). The experimental parameters chosen in the simulations are $C_{\infty}=0.012 \mathrm{~mol}, \tilde{v}_{p}=6 \times 10^{-5} \mathrm{~m} / \mathrm{s}$ and $\tilde{G}=2.3 \times 10^{3} \mathrm{~K} / \mathrm{m}$. 


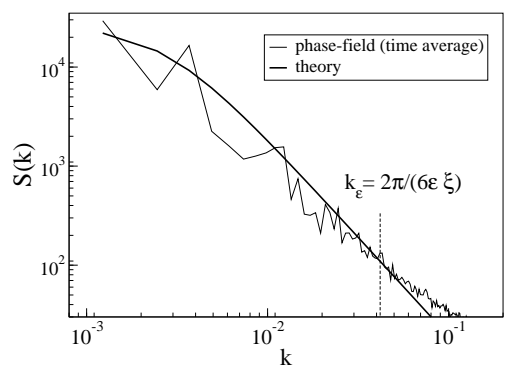

Fig 1. Mean-square interfacial fluctuation spectrum obtained from phase-field simulations and compared with the theoretical prediction.

\subsection{Stationary power spectrum}

We have simulated a planar, stationary interface in order to evaluate its spectral statistical properties. In particular, we consider the mean-square fluctuation spectrum, which can be predicted analytically by $S(k)=\int \frac{d k^{\prime}}{2 \pi}\left\langle z_{f}(k) z_{f}\left(k^{\prime}\right)\right\rangle=$ $\frac{m v_{m}}{N_{A}(1-k)\left(G+m d_{0} k^{2}\right)}$, being $z_{f}(k, t)=\int d k z_{f}(\mathbf{r}, t) e^{-i \mathbf{k r}}$ the Fourier transform of the interface position. Figure 1 compares the theoretical power spectrum $S(k)$ with the power spectrum obtained by analyzing the spectral properties of the phase-field interface. This simulation has been performed by using an explicit finite differences scheme in a $N_{x} \times N_{z}=256 \times 100$ rectangular grid with a scaled time step of $\Delta t=6.75 \times 10^{-5}$ and a scaled grid spacing $\Delta z=\Delta x=0.03$. The interface width is $\varepsilon=0.0375$, and the phase-field simulations have been thermalized during $10^{4}$ time steps before calculating any of the magnitudes presented in this work. The interfacial fluctuation spectrum has been averaged among $10^{4}$ samples taken every 10 simulation steps in order to avoid statistical time correlations. The quantitative agreement indicates the correctness of the determination of the model parameters. The scale associated to the interfacial width $\varepsilon$, which constitutes the limit of the descriptions of any phase-field model, is indicated by a vertical dashed line and determines the maximum wavelength for which the simulations results are reliable.

\subsection{Transient power spectrum and wavelength selection}

We next investigate the amplification of the fluctuations during the initial redistribution transient. The simulations are compared with a theoretical prediction based on the Warren and Langer's work on noise amplification [5]. In this reference, the time evolution of the interface correlations is described by $S(k, t)=$ $S_{0}(k) \int_{-\infty}^{t} d t^{\prime} \exp \left\{2 \int_{t^{\prime}}^{t} \omega(k, t) d t^{\prime}\right\}$ where $\omega(k, t)$ is the transient growth rate of the symmetric model [24], and $S_{0}(k)$ is given by $S_{0}(k)=\frac{\left|\omega^{e}\right| K_{B} T_{M}^{2}}{L G\left(1+d_{0}^{c} l_{T} k^{2}\right)}$, being $\omega^{e}=$ $\omega(k, t=\infty)$ the amplification rate at $t \rightarrow \infty$. Figure 2 shows the amplification of the interfacial fluctuation spectrum at three different physical times during the initial transient $(\tilde{t}=0,1.8 \mathrm{~s}$ and $2.7 \mathrm{~s})$. Dashed lines are obtained by integrating $S(k, t)$ in the adiabatic regime $\omega \ll k^{2}$. In this case the interface thickness is $\varepsilon=0.15$, and the simulations have been performed in a $500 \times 256$ grid with $\Delta z=\Delta x=0.09$, $\Delta t=0.00135$. The resulting power spectrum is noisy because the results are di- 


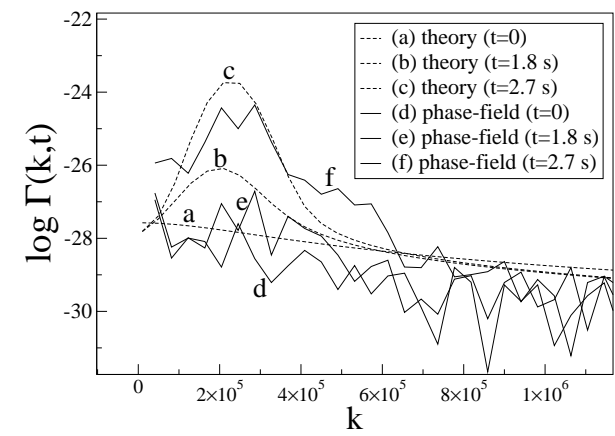

Fig 2. Noise amplification and wavelength selection during the transient. Comparison between the theoretical prediction and phase-field simulation results.

rect spectral properties of the fluctuating interface at each time and no average or filtering has been used. As it can be observed, quantitative agreement is achieved between theory and simulations in the early time amplification of fluctuations as well as in the wavelength selection of the cellular pattern. After the early stages where the growth of fluctuations is linear, the system enters in a nonlinear regime and a cellular pattern with a certain wavelength sets in the system. The end of the linear regime can be defined as the time at which the amplitude of the most unstable Fourier mode is comparable with its wavelength. This condition defines a crossover time $t_{0}$ which can be theoretically determined by the condition $\left\langle\delta z^{2}>\sim \lambda_{\max }\right.$, where $\left\langle\delta z^{2}>=\int \frac{d^{2} k}{(2 \pi)^{2}} S(k, t)\right.$ is the mean-square fluctuation amplitude and $\lambda_{\max }$ is the wavelength of the largest Fourier mode. Figure 3 shows the evolution in time of these two magnitudes in our system, and indicates the determination of the crossover time $\tilde{t}_{0}$ in physical units.

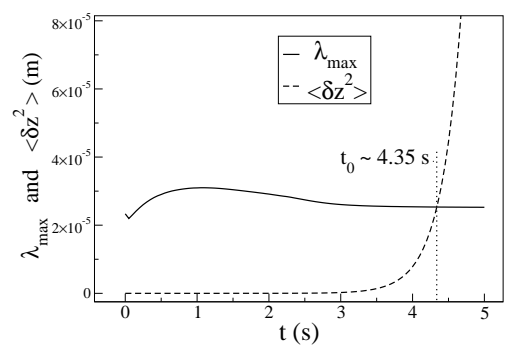

Fig 3. Determination of the crossover time $\tilde{t}_{0}$.

\section{Conclusions}

We have studied the initial transients of a directional solidification experiment of a mixture of liquid crystals in the context of the symmetric model with constant concentration gap. Contrary to previous work on transients in fluctuating phasefield simulations [24], results presented here have been obtained by using a non- 
variational formulation of the stochastic phase-field model, recently proposed [19]. Fluctuations have been implemented giving the correct equilibrium statistics but without employing any fluctuation-dissipation relation to determine the noise intensities in the model. The agreement between predictions and simulations has been excellent, thus confirming the applicability of this approach for the study of fluctuations during transient stages. As a final conclusion, the employ of this class of efficient stochastic phase-field models opens new possibilities for the quantitative study by simulation of microstructure formation in solidification processes.

\section{Acknowledgements}

This work was financially supported by Dirección General de Investigación Científica y Técnica (Spain) (Project BFM2003-07850-C03-02) and Comissionat per a Universitats i Recerca (Spain) (Project 2001/SGR/00221).

\section{References}

[1] W. J. Boettinger, S. R. Coriell, A. L. Geer, A. Karma, W. Kurz, M. Rappaz and R. Trivedi Acta. Mater. 48 (2000) 43.

[2] W. Kurz and D. Fisher Fundamentals of Solidification (Trans Tech Pub., 1989).

[3] J. Langer Rev. Mod. Phys. 52 (1980) 1.

[4] J. A. Warren and J. S. Langer Phys. Rev. A 42, 6 (1990) 3518-3525.

[5] J. A. Warren and J. S. Langer Phys. Rev. A 47, 4 (1993) 2702-2712.

[6] B. Caroli, C. Caroli and L. Ramírez-Piscina J. Crys. Growth 132 (1993) 377-388.

[7] J. M. A. Figueiredo, A. Vidal and O. N. Mesquita J. Crys. Growth 166 (1996) 222.

[8] J. M. A. Figueiredo, M. Santos, L. Ladeira and O. N. Mesquita Phys. Rev. Lett. 71, 26 (1993) 4397.

[9] R. González-Cinca, R. Folch, R. Benítez, L. Ramírez-Piscina, J. Casademunt and A. Hernández-Machado in E. Korutcheva and R. Cuerno, eds., Advances in Condensed Matter and Statistical Mechanics (Nova Science Publishers, 2004).

[10] A. Karma and W. J. Rappel Phys. Rev. E 53 (1996) R3017.

[11] A. Karma Phys. Rev. Lett. 87 (2001) 115701.

[12] R. F. Almgren SIAM J. Appl. Math. 59 (1999) 2086.

[13] A. Karma and W.-J. Rappel Phys. Rev. E 60 (1999) 3614.

[14] S. Pavlik and R. Sekerka Physica A 268 (1999) 283.

[15] S. Pavlik and R. Sekerka Physica A 277 (2000) 415.

[16] B. Halperin, P. Hohenberg and S. Ma Phys. Rev. B 10 (1974) 139.

[17] A. A. Wheeler, W. J. Boettinger and G. B. McFadden Phys. Rev. A 45 (1992) 7424.

[18] A. A. Wheeler, W. J. Boettinger and G. B. McFadden Phys. Rev. E 47 (1993) 1893.

[19] R. Benítez and L. Ramírez-Piscina submitted to Phys. Rev. Lett. (2004).

[20] W. W. Mullins and R. F. Sekerka J. Appl. Phys. 35 (1964) 444.

[21] W. Losert, D. A. Stillman, H. Z. Cummins, P. Kopczyński, W. J. Rappel and A. Karma Phys. Rev. E 58 (1998) 7492.

[22] P. Oswald, J. Bechhoefer and A. Libchaber Phys. Rev. Lett. 58, 22 (1987) 2318.

[23] A. J. Simon, J. Bechhoefer and A. Libchaber Phys. Rev. Lett. 61, 22 (1988) 2574.

[24] R. Benítez and L. Ramírez-Piscina vol. 32 of Lecture Notes in Computational Science and Engineering 160-165 (Springer-Verlag, Berlin, 2003). 